\title{
Fractional Extended and Unscented Kalman Filtering for State of Charge Estimation of Lithium-Ion Batteries
}

\author{
Martin Kupper ${ }^{1,2}$, Christopher Funk ${ }^{1}$, Marius Eckert ${ }^{1}$ and Sören Hohmann ${ }^{1}$
}

\begin{abstract}
Two state of charge estimation methods using fractional order extended and unscented Kalman filter and a nonlinear variable fractional order battery model are implemented. Both, battery model and Kalman filters are evaluated and compared using measurements of an actual lithium-ion polymer battery cell. The observability of the battery model and the influence of an initialization function on the estimation algorithms is investigated.
\end{abstract}

\section{INTRODUCTION}

The state of charge (SOC) of a battery is an important indicator of remaining run-time, e.g. in consumer electronics or electric vehicles. It is defined as the ratio of stored electric charge $Q(t)$ to rated electric charge $Q_{0}$ as in [1] by

$$
\operatorname{SOC}(t)=\frac{Q(t)}{Q_{0}} .
$$

Since the SOC can not be measured directly and to ensure reliable operation of battery-powered devices, accurate SOC estimates are required. There are a vast number of methods available for determining the SOC of a battery [1]-[3]. The most basic ones are coulomb counting and open circuit voltage (OCV) measurement. While the coulomb counting method estimates the SOC by integrating the battery's terminal current over time, the OCV measurement method exploits the nonlinear OCV-SOC characteristic of the battery [1]. Fig. 1 shows the OCV-SOC characteristic of the battery cell which is considered in this paper, exhibiting terminal current-dependent hysteresis. Due to measurement offsets and unknown initial SOC the coulomb counting method is only short-term accurate and requires correct initialization [2]. The OCV measurement does not suffer from these drawbacks but can only be performed when the battery is in a relaxed state [1]. More accurate SOC estimation methods combine current and voltage measurements e.g. using a battery model and a Kalman filter [3]. Such model based approaches retain the advantage of only requiring simple measurement equipment.

There is a growing interest in fractional order battery models based on so-called RQ chain equivalent circuits [4][7], as they provide a better approximation of a lithium-ion battery's internal impedance than traditional integer order RC chain models while using the same or a lower number of states [8] and being physically interpretable [6]. Fractional order battery models can be identified, e.g. using [8], [9].

\footnotetext{
*This work was supported by ITK Engineering.

${ }^{1}$ Martin Kupper, Christopher Funk, Marius Eckert and Sören Hohmann are with the Institute of Control Systems, Karlsruhe Institute of Technology, Karlsruhe, Germany martin.kupper@kit.edu

${ }^{2}$ Martin Kupper is also with ITK Engineering, Rülzheim, Germany
}

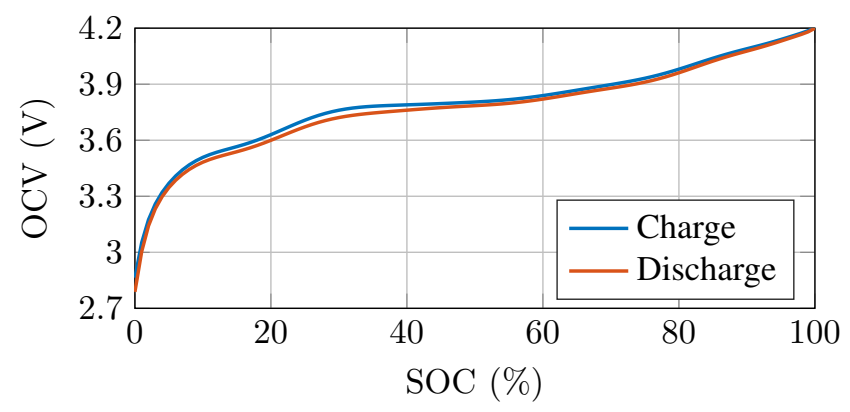

Fig. 1. OCV-SOC characteristic of a KOKAM cell of type SLPB834374H

Fractional order systems (FOS) are a class of systems described by differential equations containing fractional order derivatives i.e. generalized derivatives that allow real- and complex-valued orders of differentiation [10]. Estimating the state of FOS is a current research topic, since methods for integer order systems can not be applied directly. Hence, methods for fractional order state estimation are required. To this end the fractional order Kalman filter (FKF) and fractional order extended Kalman filter (FEKF) have been introduced in [11] and have subsequently been generalized for a broad range of systems [12]-[14]. A fractional order unscented Kalman filter (FUKF) has been investigated in [15], and in [16] an improved linear FKF that performs simultaneous estimation and smoothing, has been presented. In addition, several other state estimation methods for integer order systems have been extended to FOS, including the Luenberger observer [17], $H_{\infty}$-observers [18], [19], slidingmode observers [19], [20] and distributed and decentralized Kalman filters [21], [22].

Applications of fractional order state estimation include sensor fusion for MEMS inertial sensors [23], chaotic cryptography [24] or estimating heat conduction [17]. The FKF and FEKF have been applied to SOC estimation of battery cells employing piecewise linear [4] and nonlinear [5] fractional order models based on the RQ chain equivalent circuit. In [25] the FEKF has been applied for SOC- and current estimation of battery packs. Also, in [5] a comparison between integer and fractional order battery models and filters has been conducted, showing higher accuracy of the fractional models as well as faster convergence rates of the FEKFs. Because the advantage of fractional models and filters have already been shown in [4], [5], we do not further consider comparisons between integer and fractional order models and filters in this work. 
In this paper, we implement two SOC estimation algorithms using the FEKF, FUKF and a nonlinear variable fractional order battery model based on the RQ chain equivalent circuit and compare their performance using measurements. Due to the nonlinearities of the model the observability is investigated using the implicit function theorem. Another contribution of this paper is the investigation of the effects of a correct initialization function on the estimation process. It is shown that an exact initialization of the past states which are introduced due to the Grünwald-Letnikov definition is not necessary, since it does not improve the estimation results significantly even when the covariance matrix of the filter is chosen sufficiently small.

\section{FRACTIONAL ORDER SYSTEMS}

\section{A. Fractional Order Derivatives}

There exist several definitions of the fractional order derivative. Among these the Grünwald-Letnikov definition, the Riemann-Liouville definition and the Caputo definition are used most frequently. For the purpose of this paper, we consider a definition involving a variable fractional order $\alpha(t)$. One variation of the Grünwald-Letnikov definition for a variable fractional order derivative ${ }_{a} \mathrm{D}_{t}^{\alpha(t)}$ of an absolute continuous function $f: \mathbb{R} \rightarrow \mathbb{R}$ of time $t$ is given in [26] by

$$
{ }_{a} \mathrm{D}_{t}^{\alpha(t)} f(t)=\lim _{T \rightarrow 0} T^{-\alpha(t)} \sum_{j=0}^{\left\lfloor\frac{t-a}{T}\right\rfloor}(-1)^{j}\left(\begin{array}{c}
\alpha(t) \\
j
\end{array}\right) f(t-j T)
$$

where $a$ is the lower limit of the fractional operator, $T$ is the sampling time and $\lfloor *\rfloor$ denotes the floor function. This definition lends itself to an implementation on a computer due to its representation as a series. Note, that there exist other definitions for the fractional derivative of variable order [26]. Since the usage of other variations only barely influences the results [26], the additional computational effort of these variations is not justifiable.

\section{B. Fractional Order State-Space Representations}

The continuous-time fractional order state-space representation is similar to the integer-order case. Its state-space equations are given by

$$
\begin{aligned}
{ }_{a} \mathrm{D}_{t}^{\boldsymbol{\alpha}(t)} \boldsymbol{x}(t) & =\boldsymbol{f}(t, \boldsymbol{x}(t), \boldsymbol{u}(t))+\boldsymbol{v}(t) \\
\boldsymbol{y}(t) & =\boldsymbol{g}(t, \boldsymbol{x}(t), \boldsymbol{u}(t))+\boldsymbol{w}(t)
\end{aligned}
$$

where $\boldsymbol{u}: \mathbb{R} \rightarrow \mathbb{R}^{p}, \boldsymbol{x}: \mathbb{R} \rightarrow \mathbb{R}^{n}, \boldsymbol{v}: \mathbb{R} \rightarrow \mathbb{R}^{n}$, $\boldsymbol{y}: \mathbb{R} \rightarrow \mathbb{R}^{q}, \boldsymbol{w}: \mathbb{R} \rightarrow \mathbb{R}^{q}$ are the inputs, the states, the system noise, the outputs and the measurement noise, respectively. $\boldsymbol{f}$ are the state functions and $\boldsymbol{g}$ are the output functions. The fractional order derivatives of the states are denoted by ${ }_{a} \mathrm{D}_{t}^{\boldsymbol{\alpha}(t)} \boldsymbol{x}(t)=\left({ }_{a} \mathrm{D}_{t}^{\alpha_{1}(t)} x_{1}(t), \ldots,{ }_{a} \mathrm{D}_{t}^{\alpha_{n}(t)} x_{n}(t)\right)^{\top}$. Assuming the Grünwald-Letnikov definition in (2), $a=0$ and discrete evaluation times $t=k T, k \in \mathbb{N}$, the left-hand side of (3) can be approximated according to [27] by

$$
{ }_{0} \mathrm{D}_{t}^{\boldsymbol{\alpha}(k T)} \boldsymbol{x}(k T) \approx \boldsymbol{T}_{k}^{-1} \sum_{j=0}^{k+1}(-1)^{j} \boldsymbol{\Upsilon}_{j, k} \boldsymbol{x}((k+1-j) T)
$$

where $\boldsymbol{T}_{k}=\operatorname{diag}\left\{T^{\alpha_{1, k}}, \ldots, T^{\alpha_{n, k}}\right\}$ and $\boldsymbol{\Upsilon}_{j, k} \in \mathbb{R}^{n \times n}$ is defined as

$$
\mathbf{\Upsilon}_{j, k}=\operatorname{diag}\left\{\left(\begin{array}{c}
\alpha_{1, k} \\
j
\end{array}\right), \ldots,\left(\begin{array}{c}
\alpha_{n, k} \\
j
\end{array}\right)\right\} .
$$

Based on this approximation the discrete-time variable fractional order state-space representation

$$
\begin{aligned}
\boldsymbol{x}_{k+1} & =\boldsymbol{f}_{k}\left(\boldsymbol{x}_{k}, \boldsymbol{u}_{k}\right)+\boldsymbol{v}_{k}-\sum_{j=1}^{k+1}(-1)^{j} \boldsymbol{\Upsilon}_{j, k} \boldsymbol{x}_{k+1-j} \\
\boldsymbol{y}_{k} & =\boldsymbol{g}_{k}\left(\boldsymbol{x}_{k}, \boldsymbol{u}_{k}\right)+\boldsymbol{w}_{k}
\end{aligned}
$$

results. For reasons of concise representation the explicit dependence on $\boldsymbol{T}_{k}$ has been suppressed since $\boldsymbol{T}_{k}$ can be included in $\boldsymbol{f}_{k}$ and $\boldsymbol{v}_{k}$ [27].

\section{Initialization}

It should be noted that the states of the introduced statespace representations are not states in the classical sense, as they do not allow computation of the system's future behavior without complete knowledge of its past, up to the lower limit $a$ of the fractional order differential operator [17], [28]. This follows from

$$
{ }_{a} \mathrm{D}_{t}^{\boldsymbol{\alpha}(t)} \boldsymbol{x}(t)={ }_{b} \mathrm{D}_{t}^{\boldsymbol{\alpha}(t)} \boldsymbol{x}(t)+\boldsymbol{\Delta}^{\boldsymbol{\alpha}(t)}(t), \boldsymbol{\Delta}^{\boldsymbol{\alpha}(t)}(t) \not \equiv 0
$$

where $\boldsymbol{\Delta}^{\boldsymbol{\alpha}(t)}(t)$ is the so-called initialization function and $b>a$ [28]. Consequently one needs to consider this function in order to correctly initialize a fractional order state-space model at a time $b$ different from the modeled system's actual initial time $a$. This function is given by components in [28] by

$$
\begin{aligned}
& \Delta_{i}^{\alpha_{i}(t)}(t)= \\
& \lim _{T \rightarrow 0} T^{-\alpha_{i}(t)} \sum_{j=1}^{\left\lfloor\frac{b-a}{T}\right\rfloor}(-1)^{j+\left\lfloor\frac{t-b}{T}\right\rfloor}\left(\begin{array}{c}
\alpha_{i}(t) \\
j+\left\lfloor\frac{t-b}{T}\right\rfloor
\end{array}\right) x_{i}(b-j T) .
\end{aligned}
$$

Eq. (7) can be extended to include a sampled initialization function $\boldsymbol{\Delta}_{k}^{\boldsymbol{\alpha}_{k}}$ by using $t=k T$, (5) and (9) for the system description in (3)

$$
\boldsymbol{x}_{k+1}=\boldsymbol{f}_{k}\left(\boldsymbol{x}_{k}, \boldsymbol{u}_{k}\right)+\boldsymbol{v}_{k}-\sum_{j=1}^{k+1}(-1)^{j} \boldsymbol{\Upsilon}_{j, k} \boldsymbol{x}_{k+1-j}-\boldsymbol{\Delta}_{k}^{\boldsymbol{\alpha}_{k}}
$$

which initializes the state-space model. Since (5) is not exact, the initialization is also only approximate.

\section{VARIABLE FRACTIONAL ORDER BATTERY MODEL}

\section{A. Motivation of Fractional Order Battery Models}

Fractional order battery models can be motivated in several ways. On the one hand a derivation from basic electrochemical laws and properties as done in [6] is possible by using some simplifying assumptions. The resulting model is of fractional order and contains physically interpretable parameters, underlining the link of fractional order derivatives to physical phenomena in lithium-ion batteries. It is however, still very complex. On the other hand the use 
of fractional order battery models can be justified by the superior approximation of a lithium-ion battery's internal impedance which these models provide [8]. The internal impedance $Z$ of a linear fractional order RQ chain model consisting of an internal resistance $R_{i}$, a main capacitor $C_{0}$ and a chain of $N$ RQ circuits, i.e. $N$ RC circuits of noninteger order, is described in [9] by

$$
Z(j \omega)=R_{i}+\frac{1}{j \omega C_{0}}+\sum_{m=1}^{N} \frac{R_{m}}{1+(j \omega)^{\alpha_{m}} R_{m} Q_{m}}
$$

where $j$ is the imaginary unit, $\omega$ is the angular frequency, $R_{m}$ and $Q_{m}$ are the RQ circuits' component values and $\alpha_{m} \in \mathbb{R}^{+}$represent fractional orders of differentiation in the time domain. Note that by choosing $\alpha_{m}=1$ one obtains an integer order RC circuit. Hence the RQ chain model in (12) is a generalization of the RC chain model featuring additional parameters $\alpha_{m}$. Due to these parameters the above-mentioned superior approximation is obtained. This results in a lower number of states compared to integer order battery models, or in increased accuracy (see [4], [5]).

\section{B. Nonlinear Variable Fractional Order Battery Model}

Fig. 2 shows the nonlinear RQ chain model employed in this work. It consists of a nonlinear capacitance $C_{0}$, an internal resistance $R_{i}$ and one RQ circuit, which is comprised of the resistance $R$ and the fractional order capacitance $Q$ of order $\alpha$. The voltage-charge characteristic of $C_{0}$ is given by the battery's averaged charge and discharge OCV-SOC characteristics and is interpolated by a polynomial since the hysteresis can often be neglected for moderate and high temperatures for most lithium-ion battery types [3]. The parameters $R_{i}, R, Q$ and $\alpha$ are dependent on the SOC and are interpolated by a shape-preserving piecewise cubic function. The resulting model is then given by the SOC as in [1] by

$$
\mathrm{SOC}_{k+1}=\frac{100 T}{Q_{0}} i_{k}+\mathrm{SOC}_{k}+v_{S O C, k}
$$

and by the voltages

$$
\begin{aligned}
& u_{R Q, k+1}=\frac{T^{\alpha\left(\mathrm{SOC}_{k}\right)}}{Q\left(\mathrm{SOC}_{k}\right)}\left(i_{k}-\frac{u_{R Q, k}}{R\left(\mathrm{SOC}_{k}\right)}\right)+v_{R Q, k} \\
& -\sum_{j=1}^{k+1}(-1)^{j}\left(\begin{array}{c}
\alpha\left(\mathrm{SOC}_{k}\right) \\
j
\end{array}\right) u_{R Q, k+1-j} \\
& u_{k}=\mathrm{OCV}\left(\mathrm{SOC}_{k}\right)+u_{R Q, k}+R_{i}\left(\mathrm{SOC}_{k}\right) i_{k}+w_{k}
\end{aligned}
$$

where $u_{R Q, k}$ is defined as in Fig. $2, i_{k}$ is the battery terminal current, $T$ is the sampling period and $Q_{0}$ is the battery's rated charge. $v_{S O C, k}, v_{R Q, k}$ and $w_{k}$ are additive white gaussian noises which consist of model and measurement errors and are, inter alia, used as tuning parameters for the filters. Note that above equations are of the form of the discrete-time fractional order state-space from Sec. II-B, the first state having integer order $\alpha=1$.

The parameters of the nonlinear fractional order battery model for the lithium-ion polymer battery cell SLPB834374H from Kokam have been identified for one cell

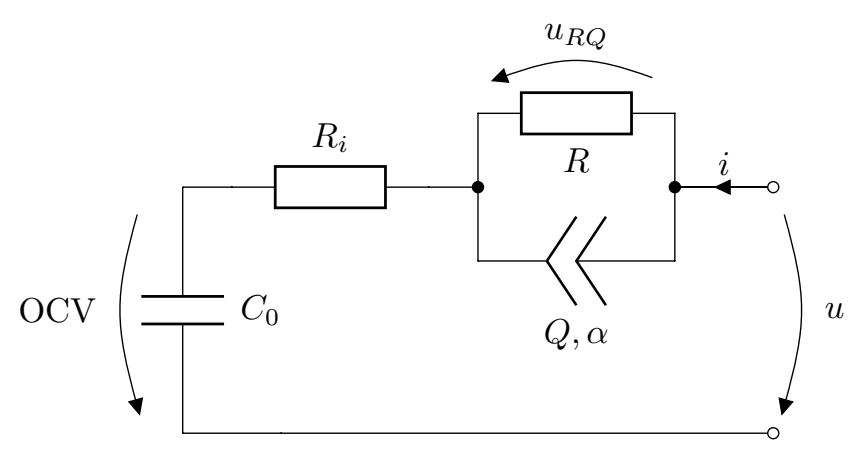

Fig. 2. 1-RQ equivalent circuit model of a battery

for several SOCs in intervals of $10 \%$, using the distribution of relaxation times (DRT) and a complex nonlinear leastsquares (CNLS) algorithm identically to [8]. The charge and discharge OCV-SOC characteristics were obtained by charging/discharging using a $C / 40=50 \mathrm{~mA}$ constant current.

\section{Battery Model Observability}

Due to the battery model's nonlinearities its observability is investigated using the implicit function theorem [29], [30]. The first two time steps of the model's output sequence $\boldsymbol{u}=\left(u_{0}, u_{1}\right)$ are calculated explicitly from an input sequence $\boldsymbol{i}=\left(i_{0}, i_{1}\right)$ and the initial state $\boldsymbol{x}_{0}=\left(S O C_{0}, u_{R Q, 0}\right)$. Based on this a continuously differentiable function

$$
\boldsymbol{q}\left(\boldsymbol{i}, \boldsymbol{u}, \boldsymbol{x}_{0}\right)=\left(\begin{array}{c}
u_{0}-q_{0}\left(\boldsymbol{i}, \boldsymbol{x}_{0}\right) \\
u_{1}-q_{1}\left(\boldsymbol{i}, \boldsymbol{x}_{0}\right)
\end{array}\right)
$$

is defined, where $u_{0}=q_{0}\left(\boldsymbol{i}, \boldsymbol{x}_{0}\right)$ and $u_{1}=q_{1}\left(\boldsymbol{i}, \boldsymbol{x}_{0}\right)$ are the calculated output values. The component functions are given by

$$
q_{0}\left(\boldsymbol{i}, \boldsymbol{x}_{0}\right)=\mathrm{OCV}\left(\mathrm{SOC}_{0}\right)+u_{R Q, 0}+R_{i} i_{0}
$$

using (15) and by

$$
\begin{aligned}
q_{1}\left(\boldsymbol{i}, \boldsymbol{x}_{0}\right)= & \operatorname{OCV}\left(\frac{100 T}{Q_{0}} i_{0}+\mathrm{SOC}_{0}\right)+\alpha\left(\mathrm{SOC}_{0}\right) u_{R Q, 0} \\
& -\frac{T^{\alpha\left(\mathrm{SOC}_{0}\right)}}{R\left(\mathrm{SOC}_{0}\right) Q\left(\mathrm{SOC}_{0}\right)} u_{R Q, 0}+\frac{T^{\alpha\left(\mathrm{SOC}_{0}\right)}}{Q\left(\mathrm{SOC}_{0}\right)} i_{0} \\
& +R_{i}\left(\frac{100 T}{Q_{0}} i_{0}+\mathrm{SOC}_{0}\right) i_{1}
\end{aligned}
$$

using (13) - (15). Then, by the implicit function theorem, if the derivative matrix

$$
\boldsymbol{Q}\left(\boldsymbol{i}, \boldsymbol{u}, \boldsymbol{x}_{0}\right)=\frac{\partial \boldsymbol{q}}{\partial \boldsymbol{x}_{0}}\left(\boldsymbol{i}, \boldsymbol{u}, \boldsymbol{x}_{0}\right)
$$

of $\boldsymbol{q}\left(\boldsymbol{i}, \boldsymbol{u}, \boldsymbol{x}_{0}\right)$ is invertible at a point $(\tilde{\boldsymbol{i}}, \tilde{\boldsymbol{u}}, \tilde{\boldsymbol{x}})$ then there is a neighborhood $V$ of $(\tilde{\boldsymbol{i}}, \tilde{\boldsymbol{u}})$ and a neighborhood $U$ of $\tilde{\boldsymbol{x}}$ such that for every $(\boldsymbol{i}, \boldsymbol{u}) \in V$ there exists exactly one $\boldsymbol{x}_{0} \in U$ satisfying $\boldsymbol{q}\left(\boldsymbol{i}, \boldsymbol{u}, \boldsymbol{x}_{0}\right)=\mathbf{0}$. That is, $\boldsymbol{x}_{0}$ can be uniquely determined from measurements. It follows that if $\boldsymbol{Q}\left(\boldsymbol{i}, \boldsymbol{u}, \boldsymbol{x}_{0}\right)$ is invertible for some $\boldsymbol{i}=\left(i_{0}, i_{1}\right)$ and all possible $\boldsymbol{x}_{0}$ then the system is locally observable. 
The condition number

$$
\zeta=\frac{\sigma_{\max }\left\{\boldsymbol{Q}\left(\boldsymbol{i}, \boldsymbol{u}, \boldsymbol{x}_{0}\right)\right\}}{\sigma_{\min }\left\{\boldsymbol{Q}\left(\boldsymbol{i}, \boldsymbol{u}, \boldsymbol{x}_{0}\right)\right\}}
$$

defined as the ratio of largest singular value $\sigma_{\max }$ to smallest singular value $\sigma_{\min }$ is a measure of the invertibility of $\boldsymbol{Q}\left(\boldsymbol{i}, \boldsymbol{u}, \boldsymbol{x}_{0}\right)$, with large values indicating a nearly singular matrix. Therefore, a large condition number indicates that large state estimation errors can occur, due to the system potentially being unobservable. Numerically evaluating the condition number of the derivative matrix at several states and for input currents in the range from $-3 \mathrm{~A}$ to $3 \mathrm{~A}$ gives an observability map which is shown logarithmically in Fig. 3. From Fig. 3 it follows that the system is locally observable except in the range of $40 \%-50 \%$ SOC, where the above (sufficient) criterion fails to determine observability. Intuitively, this result is comprehensible due to the very flat OCV-SOC characteristic in this region (see Fig. 1). For battery chemistries with a steeper OCV-SOC characteristic the observability can be expected to improve.

\section{FRACTIONAL KALMAN FILTERS}

\section{A. Fractional Order Extended Kalman Filter}

The fractional order extended Kalman filter (FEKF) [11], adapted for systems with feedthrough and time-varying fractional order, is defined by the prediction equations

$$
\begin{aligned}
\boldsymbol{x}_{k+1 \mid k}= & \boldsymbol{f}_{k}\left(\boldsymbol{x}_{k \mid k}, \boldsymbol{u}_{k}\right)-\sum_{j=1}^{k+1}(-1)^{j} \boldsymbol{\Upsilon}_{j, k} \boldsymbol{x}_{k+1-j \mid k+1-j} \\
\boldsymbol{P}_{k+1 \mid k}^{\boldsymbol{x} \boldsymbol{x}}= & \left(\boldsymbol{F}_{k}+\boldsymbol{\Upsilon}_{1, k}\right) \boldsymbol{P}_{k \mid k}^{\boldsymbol{x} \boldsymbol{x}}\left(\boldsymbol{F}_{k}+\boldsymbol{\Upsilon}_{1, k}\right)^{\top}+\boldsymbol{Q}_{k} \\
& +\sum_{j=2}^{k+1} \boldsymbol{\Upsilon}_{j, k} \boldsymbol{P}_{k+1-j \mid k+1-j}^{\boldsymbol{x} \boldsymbol{x}} \boldsymbol{\Upsilon}_{j, k}^{\top}
\end{aligned}
$$

the update equations

$$
\begin{aligned}
\boldsymbol{x}_{k+1 \mid k+1}= & \boldsymbol{x}_{k+1 \mid k} \\
& +\boldsymbol{K}_{k+1}\left(\boldsymbol{y}_{k+1}-\boldsymbol{g}_{k+1}\left(\boldsymbol{x}_{k+1 \mid k}, \boldsymbol{u}_{k+1}\right)\right) \\
\boldsymbol{P}_{k+1 \mid k+1}^{\boldsymbol{x x}}= & \left(\boldsymbol{I}-\boldsymbol{K}_{k+1} \boldsymbol{G}_{k+1}\right) \boldsymbol{P}_{k+1 \mid k}^{\boldsymbol{x x}}\left(\boldsymbol{I}-\boldsymbol{K}_{k+1} \boldsymbol{G}_{k+1}\right)^{\top} \\
& +\boldsymbol{K}_{k+1} \boldsymbol{R}_{k+1} \boldsymbol{K}_{k+1}^{\top},
\end{aligned}
$$

and the Kalman gain matrix

$$
\boldsymbol{K}_{k+1}=\boldsymbol{P}_{k+1 \mid k}^{\boldsymbol{x} \boldsymbol{x}} \boldsymbol{G}_{k+1}^{\top}\left(\boldsymbol{G}_{k+1} \boldsymbol{P}_{k+1 \mid k}^{\boldsymbol{x} \boldsymbol{x}} \boldsymbol{G}_{k+1}^{\top}+\boldsymbol{R}_{k+1}\right)^{-1}
$$

where $\boldsymbol{P}_{k+1 \mid k}^{\boldsymbol{x} \boldsymbol{x}}, \boldsymbol{P}_{k+1 \mid k+1}^{\boldsymbol{x} \boldsymbol{x}}, \boldsymbol{R}_{k}, \boldsymbol{Q}_{k}$ are the predicted/updated estimation error covariance matrices and the system and measurement noise covariance matrices, respectively. Matrices $\boldsymbol{F}_{k}$ and $\boldsymbol{G}_{k+1}$ are given by

$$
\begin{aligned}
\boldsymbol{F}_{k} & =\left[\frac{\partial \boldsymbol{f}_{k}\left(\boldsymbol{x}_{k}, \boldsymbol{u}_{k}\right)}{\partial \boldsymbol{x}_{k}}\right]_{\boldsymbol{x}_{k}=\boldsymbol{x}_{k \mid k}} \\
\boldsymbol{G}_{k+1} & =\left[\frac{\partial \boldsymbol{g}_{k+1}\left(\boldsymbol{x}_{k+1}, \boldsymbol{u}_{k+1}\right)}{\partial \boldsymbol{x}_{k+1}}\right]_{\boldsymbol{x}_{k+1}=\boldsymbol{x}_{k+1 \mid k}} .
\end{aligned}
$$

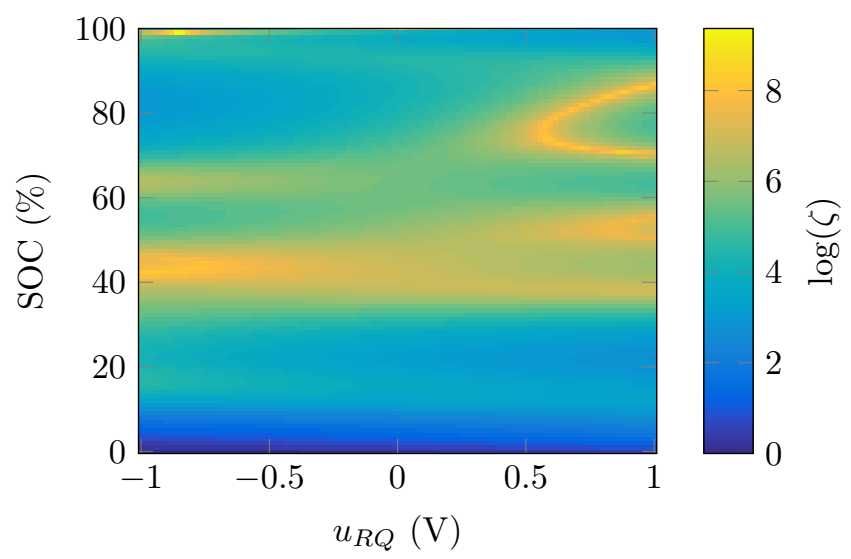

Fig. 3. Map of condition number $\log (\zeta)$ of the observability matrix of the nonlinear variable fractional order battery model

The FEKF is derived under the assumption, that the system states at different time steps are uncorrelated [11]. Nonlinearities are treated by linearization, which introduces an additional error. Due to these assumptions and approximations the FEKF is not a minimum mean square error (MMSE) optimal estimator. Nonetheless, in [11] it was shown that the FEKF can achieve satisfactory results.

\section{B. Fractional Order Unscented Kalman Filter}

The fractional order unscented Kalman filter (FUKF) [15], again adapted for systems with feedthrough and time-varying fractional order, is defined by the prediction equations

$$
\begin{aligned}
\boldsymbol{x}_{k+1 \mid k}= & \boldsymbol{f}_{k+1 \mid k}-\sum_{j=1}^{k+1}(-1)^{j} \boldsymbol{\Upsilon}_{j, k} \boldsymbol{x}_{k+1-j \mid k+1-j} \\
\boldsymbol{P}_{k+1 \mid k}^{\boldsymbol{x} \boldsymbol{x}}= & \boldsymbol{P}_{k+1 \mid k}^{\boldsymbol{f f}}+\boldsymbol{\Upsilon}_{1, k} \boldsymbol{P}_{k+1 \mid k}^{\boldsymbol{x f}}+\left(\boldsymbol{P}_{k+1 \mid k}^{\boldsymbol{x} \boldsymbol{f}}\right)^{\top} \boldsymbol{\Upsilon}_{1, k}^{\top} \\
& +\boldsymbol{Q}_{k}+\sum_{j=1}^{k+1} \boldsymbol{\Upsilon}_{j, k} \boldsymbol{P}_{k+1-j \mid k+1-j}^{\boldsymbol{x} \boldsymbol{x}} \boldsymbol{\Upsilon}_{j, k}^{\top}
\end{aligned}
$$

the update equations

$$
\begin{aligned}
& \boldsymbol{x}_{k+1 \mid k+1}=\boldsymbol{x}_{k+1 \mid k}+\boldsymbol{K}_{k+1}\left(\boldsymbol{y}_{k+1}-\boldsymbol{g}_{k+1 \mid k}\right) \\
& \boldsymbol{P}_{k+1 \mid k+1}^{\boldsymbol{x} \boldsymbol{x}}=\boldsymbol{P}_{k+1 \mid k}^{\boldsymbol{x} \boldsymbol{x}}-\boldsymbol{K}_{k+1}\left(\boldsymbol{P}_{k+1 \mid k}^{\boldsymbol{g g}}+\boldsymbol{R}_{k+1}\right) \boldsymbol{K}_{k+1}^{\top},
\end{aligned}
$$

and the Kalman gain matrix

$$
\boldsymbol{K}_{k+1}=\boldsymbol{P}_{k+1 \mid k}^{\boldsymbol{x g}}\left(\boldsymbol{P}_{k+1 \mid k}^{\boldsymbol{g g}}+\boldsymbol{R}_{k+1}\right)^{-1}
$$

The unscented transformation is used to calculate $\boldsymbol{f}_{k+1 \mid k}$ and $\boldsymbol{g}_{k+1 \mid k}$ as well as $\boldsymbol{P}_{k+1 \mid k}^{\boldsymbol{f f}}, \boldsymbol{P}_{k+1 \mid k}^{\boldsymbol{x} \boldsymbol{f}}, \boldsymbol{P}_{k+1 \mid k}^{\boldsymbol{g g}}$ and $\boldsymbol{P}_{k+1 \mid k}^{\boldsymbol{x g}}$. To this end deterministic samples are generated

$$
\boldsymbol{\chi}_{i}= \begin{cases}\boldsymbol{x}_{k \mid k} & i=0 \\ \boldsymbol{x}_{k \mid k}+\left(\sqrt{(n+\kappa) \boldsymbol{P}_{k \mid k}^{\boldsymbol{x} \boldsymbol{x}}}\right)_{i} & i=1 \ldots n \\ \boldsymbol{x}_{k \mid k}-\left(\sqrt{(n+\kappa) \boldsymbol{P}_{k \mid k}^{\boldsymbol{x} \boldsymbol{x}}}\right)_{i-n} & i=n+1 \ldots 2 n\end{cases}
$$




$$
\boldsymbol{\psi}_{i}= \begin{cases}\boldsymbol{x}_{k+1 \mid k} & i=0 \\ \boldsymbol{x}_{k+1 \mid k}+\left(\sqrt{(n+\kappa) \boldsymbol{P}_{k+1 \mid k}^{\boldsymbol{x}}}\right)_{i} & i=1 \ldots n \\ \boldsymbol{x}_{k+1 \mid k}-\left(\sqrt{(n+\kappa) \boldsymbol{P}_{k+1 \mid k}^{\boldsymbol{x}}}\right)_{i-n} & i=n+1 \ldots 2 n\end{cases}
$$

where $n$ is the state-space's dimension and $\kappa$ is a tuning parameter determining sample spread. Based on the samples $\chi_{i}$ and $\psi_{i}$ the unknowns are calculated as follows:

$$
\begin{aligned}
\boldsymbol{f}_{k+1 \mid k}= & \sum_{i=0}^{2 n} w_{i} \boldsymbol{f}_{k}\left(\boldsymbol{\chi}_{i}, \boldsymbol{u}_{k}\right) \\
\boldsymbol{P}_{k+1 \mid k}^{\boldsymbol{f f}}= & \sum_{i=0}^{2 n}\left[w_{i}\left(\boldsymbol{f}_{k}\left(\boldsymbol{\chi}_{i}, \boldsymbol{u}_{k}\right)-\boldsymbol{f}_{k+1 \mid k}\right)\right. \\
& \left.\cdot\left(\boldsymbol{f}_{k}\left(\boldsymbol{\chi}_{i}, \boldsymbol{u}_{k}\right)-\boldsymbol{f}_{k+1 \mid k}\right)^{\top}\right] \\
\boldsymbol{P}_{k+1 \mid k}^{\boldsymbol{x f}}= & \sum_{i=0}^{2 n} w_{i}\left(\boldsymbol{\chi}_{i}-\boldsymbol{x}_{k \mid k}\right)\left(\boldsymbol{f}_{k}\left(\boldsymbol{\chi}_{i}, \boldsymbol{u}_{k}\right)-\boldsymbol{f}_{k+1 \mid k}\right)^{\top} \\
\boldsymbol{g}_{k+1 \mid k}= & \sum_{i=0}^{2 n} w_{i} \boldsymbol{g}_{k+1}\left(\boldsymbol{\psi}_{i}, \boldsymbol{u}_{k+1}\right) \\
\boldsymbol{P}_{k+1 \mid k}^{\boldsymbol{g g}}= & \sum_{i=0}^{2 n}\left[w_{i}\left(\boldsymbol{g}_{k+1}\left(\boldsymbol{\psi}_{i}, \boldsymbol{u}_{k+1}\right)-\boldsymbol{g}_{k+1 \mid k}\right)\right. \\
& \left.\cdot\left(\boldsymbol{g}_{k+1}\left(\boldsymbol{\psi}_{i}, \boldsymbol{u}_{k+1}\right)-\boldsymbol{g}_{k+1 \mid k}\right)^{\top}\right] \\
\boldsymbol{P}_{k+1 \mid k}^{\boldsymbol{x g}}= & \sum_{i=0}^{2 n}\left[w_{i}\left(\boldsymbol{\psi}_{i}-\boldsymbol{x}_{k+1 \mid k}\right)\right. \\
& \left.\cdot\left(\boldsymbol{g}_{k+1}\left(\boldsymbol{\psi}_{i}, \boldsymbol{u}_{k+1}\right)-\boldsymbol{g}_{k+1 \mid k}\right)^{\top}\right]
\end{aligned}
$$

where $w_{i}$ are weights defined by

$$
w_{i}=\left\{\begin{array}{ll}
\frac{\kappa}{n+\kappa} & i=0 \\
\frac{1}{2(n+\kappa)} & i=1 \ldots 2 n
\end{array} .\right.
$$

As with the FEKF it is assumed that the system states at different time steps are uncorrelated, resulting in a suboptimal estimator. By using the unscented transformation instead of linearization a better approximation of the system's nonlinearities can be achieved and linearization errors can be reduced [31].

\section{Short Memory Principle}

The algorithms presented in sections IV-A and IV-B are not directly suited for implementation on a computer. This is due to their unbounded memory requirements: As time progresses more and more estimates have to be saved to compute the prediction equations. However, as the elements of $\boldsymbol{\Upsilon}_{j, k}$ converge to zero for $j \rightarrow \infty$ [32], very old estimates can be neglected in the prediction equations. Thus, only a fixed number $L$, where $L$ is called memory length, of previous estimates have to be saved. Employing this simplification, which is known as short memory principle, the algorithms can be implemented on a computer using finite memory [27].

\section{Initialization}

In accord with Sec. II-C the FEKF and FUKF can be adapted to incorporate the initialization function $\boldsymbol{\Delta}_{k}^{\boldsymbol{\alpha}_{k}}$. As a matter of fact, only the state prediction equations need to be modified. This results in the following equation for the FEKF

$$
\begin{aligned}
\boldsymbol{x}_{k+1 \mid k}= & \boldsymbol{f}_{k}\left(\boldsymbol{x}_{k \mid k}, \boldsymbol{u}_{k}\right)-\sum_{j=1}^{k+1}(-1)^{j} \boldsymbol{\Upsilon}_{j, k} \boldsymbol{x}_{k+1-j \mid k+1-j} \\
& -\boldsymbol{\Delta}_{k}^{\boldsymbol{\alpha}_{k}}
\end{aligned}
$$

and

$$
\boldsymbol{x}_{k+1 \mid k}=\boldsymbol{f}_{k+1 \mid k}-\sum_{j=1}^{k+1}(-1)^{j} \boldsymbol{\Upsilon}_{j, k} \boldsymbol{x}_{k+1-j \mid k+1-j}-\boldsymbol{\Delta}_{k}^{\boldsymbol{\alpha}_{k}}
$$

for the FUKF, where all symbols are defined as before.

\section{MEASUREMENTS}

In order to validate the nonlinear fractional order battery model and the fractional order Kalman filters, voltage and current measurements of a battery cell SLPB $834374 \mathrm{H}$ from Kokam were taken using the setup shown in Fig. 4. The setup contains a DS2004 A/D-board from dSpace, a BOP2020M current source from Kepco and a highly accurate (max. $0.12 \%$ current measurement error) 34410A multimeter from Agilent Technologies. Note that we use two different amperemeters for comparison reasons which is explained later in Sec. VI-B. The battery was loaded according to the current profile shown in Fig. 5 and both, current and terminal voltage, were measured. The sampling periods were chosen to be $0.1 \mathrm{~s}$ for the DS2004 A/D-board and $0.01 \mathrm{~s}$ for the 34410A multimeter. Before applying the current profile to the battery an initial OCV measurement was taken in order to determine the initial SOC using the OCV-SOC relation.

\section{RESULTS AND DISCUSSION}

\section{A. Validation of the Battery Model in Time-Domain}

Based on the proposed battery model and the validation current profile shown in Fig. 5, simulations were conducted.

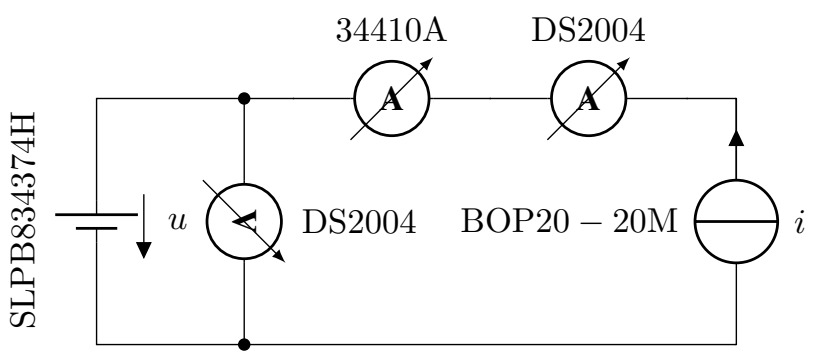

Fig. 4. Measurement setup 


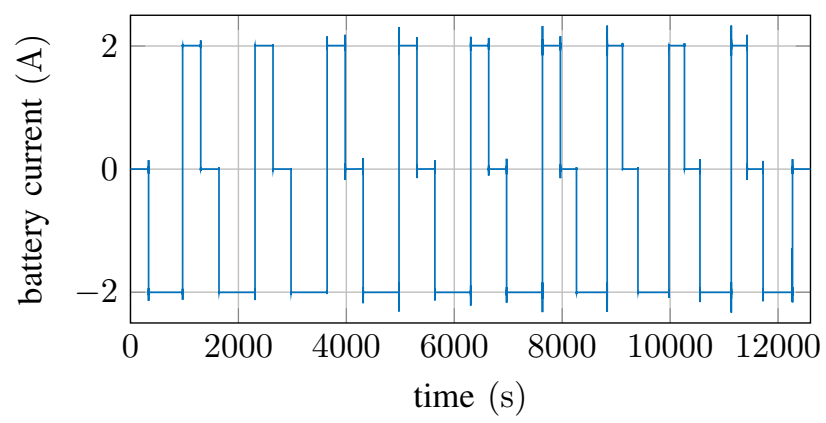

Fig. 5. Validation current profile measured by the 34410A multimeter

The results were validated using the terminal voltage measurements from Sec. V. The initial values of the simulations were set to

$$
\boldsymbol{x}_{0}=\left(\begin{array}{c}
100 \% \\
0 \mathrm{~V}
\end{array}\right)
$$

where the initial SOC was determined using the initial OCV measurement described in Sec. V. Fig. 6 shows simulated and measured battery voltage in comparison using a memory length of $L=1000$ and a simulation stepsize of $T=0.1 \mathrm{~s}$. Root-mean-square error (RMSE) and maximum absolute error (MAE) are shown in Tab. I for several memory lengths. The results demonstrate that for large memory lengths the battery model is very accurate, achieving a RMSE of $0.0204 \mathrm{~V}$ for $L=1000$. Even for lower memory lengths the results are satisfactory. For all cases, the maximum error is attained at the end of the simulation. The reason is that a small deviation $\triangle \mathrm{SOC}$ results in a comparatively large $\triangle \mathrm{OCV}$ because of the steep OCV-SOC characteristic at low SOCs.

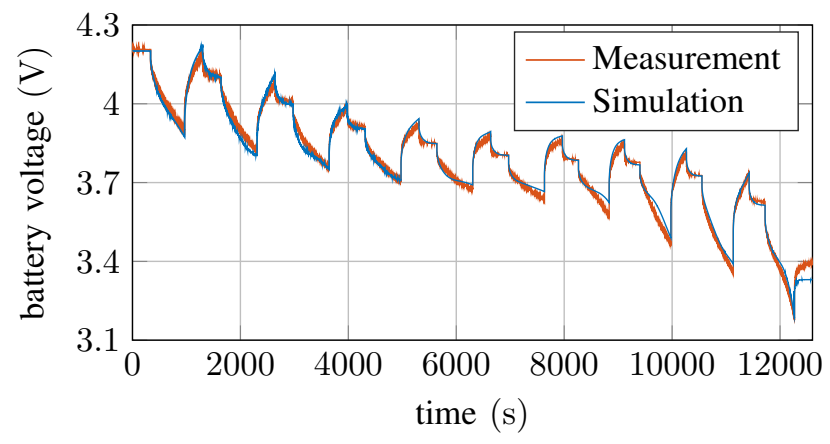

Fig. 6. Comparison of simulated and measured battery voltage $(L=1000)$

TABLE I

BATTERY VOLTAGE SIMULATION ERROR

\begin{tabular}{ccc}
\hline$L$ & RMSE & MAE \\
\hline 25 & $0.0390 \mathrm{~V}$ & $0.1121 \mathrm{~V}$ \\
100 & $0.0279 \mathrm{~V}$ & $0.0950 \mathrm{~V}$ \\
250 & $0.0218 \mathrm{~V}$ & $0.0830 \mathrm{~V}$ \\
1000 & $0.0204 \mathrm{~V}$ & $0.0701 \mathrm{~V}$ \\
\hline
\end{tabular}

\section{B. Validation of Fractional Order Kalman Filters}

The measurements from Sec. V were used to validate the FEKF and FUKF and to generate a SOC reference by coulomb counting. The fractional order Kalman filters received measurement data from the DS2004 A/D-board, whereas the coulomb counting algorithm used measurement data provided by the highly accurate Agilent 34410A amperemeter. The coulomb counting algorithm was initialized using (44). The FEKF's and FUKF's initial estimation error covariance matrices as well as the system noise covariance matrices were determined by trial and error and were set to

$$
\begin{aligned}
\boldsymbol{P}_{0 \mid 0} & =\left(\begin{array}{cc}
1 \%^{2} & 0 \\
0 & 10 \mathrm{~V}^{2}
\end{array}\right) \\
\boldsymbol{Q}_{F E K F}=\boldsymbol{Q}_{F U K F} & =\left(\begin{array}{cc}
10^{-5} \%^{2} & 0 \\
0 & 8 \cdot 10^{-5} \mathrm{~V}^{2}
\end{array}\right) .
\end{aligned}
$$

The measurement noise covariance matrices were computed from voltage measurements in steady state:

$$
\boldsymbol{R}_{F E K F}=\boldsymbol{R}_{F U K F}=2.8391 \cdot 10^{-8} \mathrm{~V}^{2} .
$$

A tuning parameter $\kappa=1$ was selected for the FUKF. First, the correct initial values for FEKF and FUKF as in (44)

$$
\boldsymbol{x}_{0 \mid 0}=\left(\begin{array}{c}
100 \% \\
0 \mathrm{~V}
\end{array}\right)
$$

according to the initial OCV measurement from Sec. V has been chosen. Fig. 7 shows the estimated SOC in comparison to the reference for a memory length of $L=250$. The estimation error's dependence on the memory length is outlined in Tab. II. Both the FEKF and the FUKF provide good estimates of the SOC for SOC $>40 \%$. Below this threshold their estimates exhibit deviations from the reference. This is caused by the flat OCV-SOC characteristic around SOC $=40 \%$ (see Fig. 1) which causes poor observability as discussed in Sec. III-C. In Fig. 7 also the estimation error covariance of the SOC is given which is the upper left element of $\boldsymbol{P}_{k \mid k}$. It can be seen that the uncertainty of the filters is consistent with the actual errors and the poor observability. The FUKF exhibits only a slightly better estimation performance compared to the FEKF. The RMSE and the MAE of both algorithms can be reduced significantly, at the cost of additional computational expense by increasing the memory length $L$ (see Tab. II).

In a second approach, the same measurement data have been taken but both filters FEKF and FUKF use wrong initial values:

$$
\boldsymbol{x}_{0 \mid 0}=\left(\begin{array}{c}
90 \% \\
0 \mathrm{~V}
\end{array}\right) \text {. }
$$

The resulting RMSE of the algorithms is also shown in Tab. II. It can be seen that both filters can handle the wrong starting SOC, resulting only in a slightly higher RMSE compared to the correctly initialized filters.

\section{Influence of an Initialization Function}

The effect of an initialization function on the state estimation was examined using a simulation of the step-shaped current profile in Fig. 8. The estimation algorithms were 


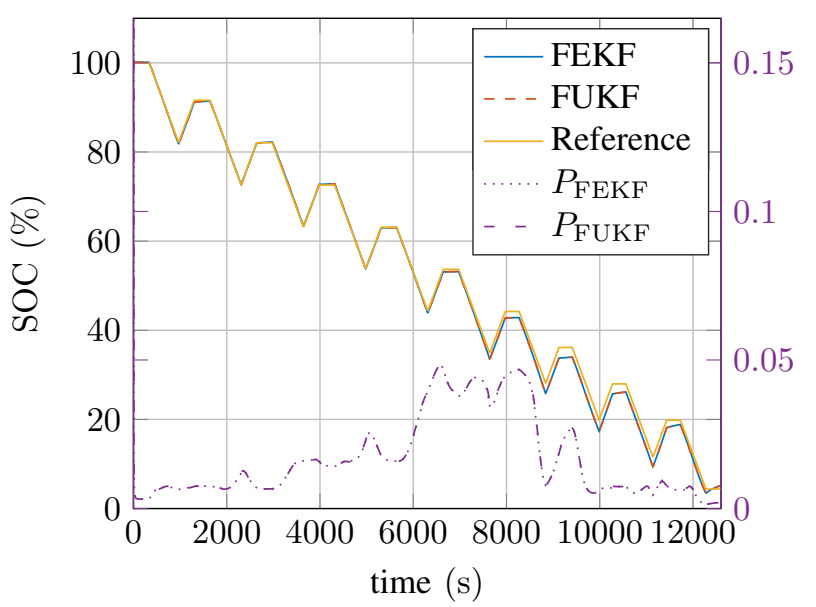

Fig. 7. SOC estimation using measurement data and upper left element of the covariance $\boldsymbol{P}_{k \mid k}$ for both estimators $(L=250)$

TABLE II

COMPARISON OF SOC ESTIMATION ERRORS

\begin{tabular}{cc|cc|c}
\hline \multirow{2}{*}{ Algorithm } & $L$ & \multicolumn{2}{|c|}{ Correct $\boldsymbol{x}_{0 \mid 0}$} & Wrong $\boldsymbol{x}_{0 \mid 0}$ \\
& RMSE & MAE & RMSE \\
\hline \multirow{4}{*}{ FEKF } & 25 & $3.0092 \%$ & $6.8703 \%$ & $3.0752 \%$ \\
& 100 & $1.7889 \%$ & $3.9161 \%$ & $2.1359 \%$ \\
& 250 & $1.1846 \%$ & $2.6129 \%$ & $1.8592 \%$ \\
& 1000 & $0.7239 \%$ & $1.4371 \%$ & $1.8029 \%$ \\
\hline \multirow{4}{*}{ FUKF } & 25 & $3.0029 \%$ & $6.8608 \%$ & $3.0695 \%$ \\
& 100 & $1.7852 \%$ & $3.9114 \%$ & $2.1343 \%$ \\
& 250 & $1.1838 \%$ & $2.6123 \%$ & $1.8590 \%$ \\
& 1000 & $0.7239 \%$ & $1.4370 \%$ & $1.8028 \%$ \\
\hline
\end{tabular}

started after the current step at $t_{0}=1800 \mathrm{~s}$, in order to obtain an initialized fractional order battery model. The simulations' initial values were set to $\mathrm{SOC}_{0}=30 \%$ and $u_{R Q, 0}=0 \mathrm{~V}$. A memory length of $L=1000$ was chosen to get an accurate simulation. After a simulated time of $t_{0}=1800 \mathrm{~s}$ the state $\mathrm{SOC}=82.361 \%$ and $u_{R Q}=0.089 \mathrm{~V}$ was reached. The initialization function is calculated using (10) with $a=0, b=t_{0}, T=0.1 \mathrm{~s}$ resulting in $\Delta_{S O C}^{1}=0$ and

$\Delta_{R Q}^{\alpha_{k}}=T^{-\alpha_{k}} \sum_{j=1}^{18000}(-1)^{j+k}\left(\begin{array}{c}\alpha_{k} \\ j+k-18000\end{array}\right) u_{R Q, 18000-j}$.

The FEKF and FUKF were initialized using

$$
\begin{aligned}
\boldsymbol{x}_{0 \mid 0} & =\left(\begin{array}{c}
82.361 \% \\
0.089 \mathrm{~V}
\end{array}\right), \\
\boldsymbol{P}_{0 \mid 0} & =\left(\begin{array}{cc}
10^{-6 \% \%^{2}} & 0 \\
0 & 10^{-6} V^{2}
\end{array}\right)
\end{aligned}
$$

and the measurement and system noise covariance matrices were chosen identically to VI-B. A memory length of $L=1000$ was employed. Note that the covariance $\boldsymbol{P}_{0 \mid 0}$ in (52) has been chosen significantly smaller than the covariance before in (45). Also note that the filters without

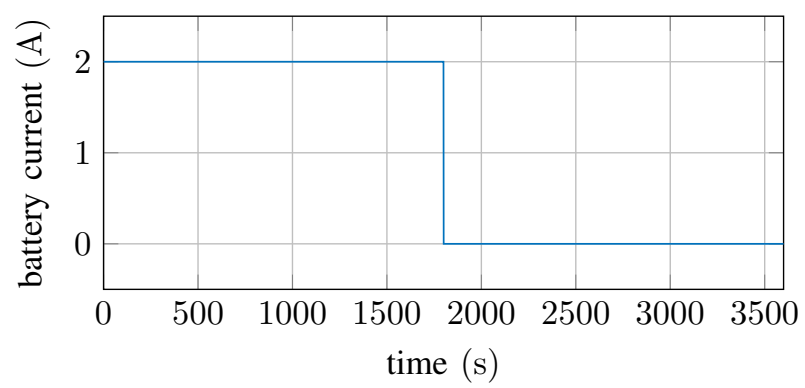

Fig. 8. Current step profile for the simulation of an initialized battery

initialization use (21) and (28) for the prediction as before, and the filters with initialization use (42) and (43), respectively. Fig. 9 shows the results of the state estimation using the FEKF and FUKF with and without initialization function. It can be seen that the initialization function has negligible influence on the estimation results. The reason for this is the dominating influence of the noise covariance matrices and the estimation error covariance matrix $\boldsymbol{P}_{k \mid k}$ even though we chose a clearly smaller initial covariance $\boldsymbol{P}_{0 \mid 0}$ in (52). In addition to that, the resulting estimation error in this example is at all times in all cases smaller than 0.04 percentage points which is insignificant, especially considering increased noises and that one does not know the exact initial SOC in practice. Therefore, the initialization function can be disregarded without any problems for this application example.

\section{CONCLUSIONS}

In this paper two SOC estimation methods based on the FEKF and FUKF and a nonlinear variable fractional order battery model have been proposed and compared. Since the FEKF and the FUKF differ only in the prediction step from its integer order equivalents, the additional calculation power and memory demand of the fractional calculus is confined to the calculation of the sum induced by the Grünwald-Letnikov definition in dependency on the memory length $L$.

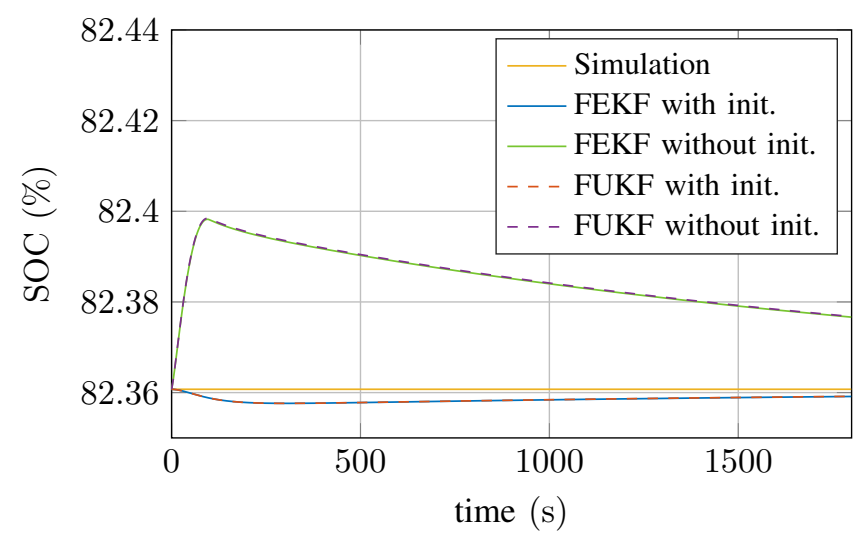

Fig. 9. SOC estimates of the simulation with and without initialization function 
The battery model has been shown to be locally observable except for a region with flat OCV-SOC characteristic. The SOC estimators and the battery model have been validated by measurements. The results show that both methods produce very accurate SOC estimates except where the OCV-SOC characteristic is flat which matches with the observability analysis. The estimates in this region are less accurate but still acceptable. The overall estimation performance of both proposed methods is nearly identical, because the considered nonlinearities are weak and linearization errors are negligible. Nevertheless, for more complex models which consider temperature- and current-dependencies it is expected that the FUKF provides better results.

Moreover, it could be seen that the influence of an initialization function is negligible compared to the impact of noises and covariance matrices, especially considering that prior knowledge about the initial states and the initialization function of the system is normally unknown.

\section{REFERENCES}

[1] W.-Y. Chang, "The State of Charge Estimating Methods for Battery: A Review," ISRN Applied Mathematics, pp. 1-7, 2013.

[2] M. Coleman, C. K. Lee, C. Zhu, and W. Hurley, "State-of-Charge Determination From EMF Voltage Estimation: Using Impedance, Terminal Voltage, and Current for Lead-Acid and Lithium-Ion Batteries," IEEE Transactions on Industrial Electronics, vol. 54, no. 5, pp. 25502557, Oct. 2007.

[3] W. Waag, C. Fleischer, and D. U. Sauer, "Critical review of the methods for monitoring of lithium-ion batteries in electric and hybrid vehicles," Journal of Power Sources, vol. 258, pp. 321-339, July 2014.

[4] J. Xu, C. C. Mi, B. Cao, and J. Cao, "A new method to estimate the state of charge of lithium-ion batteries based on the battery impedance model," Journal of Power Sources, vol. 233, pp. 277-284, July 2013.

[5] R. Xiao, J. Shen, X. Li, W. Yan, E. Pan, and Z. Chen, "Comparisons of Modeling and State of Charge Estimation for Lithium-Ion Battery Based on Fractional Order and Integral Order Methods," Energies, vol. 9, no. 3, p. 184, Mar. 2016.

[6] J. Sabatier, M. Merveillaut, J. M. Francisco, F. Guillemard, and D. Porcelatto, "Fractional models for lithium-ion batteries," in 2013 European Control Conference (ECC), July 2013, pp. 3458-3463.

[7] J. Sabatier, M. Merveillaut, J. M. Francisco, F. Guillemard, and D. Porcelatto, "Lithium-ion batteries modeling involving fractional differentiation," Journal of Power Sources, vol. 262, pp. 36-43, Sept. 2014.

[8] M. Eckert, M. Kupper, and S. Hohmann, "Functional Fractional Calculus for System Identification of Battery Cells," at - Automatisierungstechnik, vol. 62, no. 4, Jan. 2014.

[9] M. Eckert, L. Kölsch, and S. Hohmann, "Fractional algebraic identification of the distribution of relaxation times of battery cells," in 54th IEEE Conference on Decision and Control (CDC). IEEE, Dec. 2015, pp. 2101-2108.

[10] I. Podlubny, Fractional differential equations: an introduction to fractional derivatives, fractional differential equations, to methods of their solution and some of their applications, ser. Mathematics in science and engineering. San Diego: Academic Press, 1999, no. 198.

[11] D. Sierociuk and A. Dzieliński, "Fractional kalman filter algorithm for the states, parameters and order of fractional system estimation," International Journal of Applied Mathematics and Computer Science, vol. 16, no. 1, pp. 129-140, 2006.

[12] D. Sierociuk and P. Ziubinski, "Fractional Order Estimation Schemes for Fractional and Integer Order Systems with Constant and Variable Fractional Order Colored Noise," Circuits, Systems, and Signal Processing, vol. 33, no. 12, pp. 3861-3882, Dec. 2014.
[13] D. Sierociuk, "Fractional Kalman Filter algorithms for correlated system and measurement noises," Control and Cybernetics, vol. 42, no. 2, 2013.

[14] D. Sierociuk and P. Ziubinski, "Variable Order Fractional Kalman Filters for Estimation over Lossy Network," in Advances in Modelling and Control of Non-integer-Order Systems, K. J. Latawiec, M. Lukaniszyn, and R. Stanislawski, Eds. Cham: Springer International Publishing, 2015, vol. 320, pp. 285-294.

[15] R. Caballero-Aguila, A. Hermoso-Carazo, and J. Linares-Perez, "Extended and unscented filtering algorithms in nonlinear fractional order systems with uncertain observations," Appl. Math. Sci, vol. 6, no. 2932, pp. 1471-1486, 2012.

[16] D. Sierociuk, I. Tejado, and B. M. Vinagre, "Improved fractional Kalman filter and its application to estimation over lossy networks," Signal Processing, vol. 91, no. 3, pp. 542-552, Mar. 2011.

[17] J. Sabatier, C. Farges, M. Merveillaut, and L. Feneteau, "On Observability and Pseudo State Estimation of Fractional Order Systems," European Journal of Control, vol. 18, no. 3, pp. 260-271, Jan. 2012.

[18] Y. Boukal, M. Darouach, M. Zasadzinski, and N. Radhy, "H $\infty$ observer design for linear fractional-order systems in time and frequency domains," in 2014 European Control Conference (ECC). IEEE, June 2014, pp. 2975-2980.

[19] S.-C. Lee, Y. Li, Y. Chen, and H.-S. Ahn, "Hळ and Sliding Mode Observers for Linear Time-Invariant Fractional-Order Dynamic Systems With Initial Memory Effect," Journal of Dynamic Systems, Measurement, and Control, vol. 136, no. 5, p. 051022, July 2014.

[20] N. Djeghali, S. Djennoune, M. Bettayeb, M. Ghanes, and J.-P. Barbot, "Observation and sliding mode observer for nonlinear fractional-order system with unknown input," ISA Transactions, vol. 63, pp. 1-10, July 2016.

[21] M. Kupper, I. Sesar Gil, and S. Hohmann, "Distributed and decentralized state estimation of Fractional order systems," in 2016 American Control Conference (ACC). IEEE, July 2016, pp. 2765-2771.

[22] M. Kupper, I. Sesar Gil, and S. Hohmann, "Distributed and decentralized kalman filtering for cascaded fractional order systems," in 2017 American Control Conference (ACC). IEEE, 2017, pp. 5223-5230.

[23] M. Romanovas, L. Klingbeil, M. Traechtler, and Y. Manoli, "Application of fractional sensor fusion algorithms for inertial mems sensing," Mathematical Modelling and Analysis, vol. 14, no. 2, pp. 199-209, Jan. 2009.

[24] H. Sadeghian, H. Salarieh, A. Alasty, and A. Meghdari, "On the fractional-order extended Kalman filter and its application to chaotic cryptography in noisy environment," Applied Mathematical Modelling, vol. 38, no. 3, pp. 961-973, Feb. 2014.

[25] M. Kupper, J. Brenneisen, O. Stark, S. Krebs, and S. Hohmann, "Cascaded fractional kalman filtering for state and current estimation of large-scale lithium-ion battery packs," in 30th Chinese Control And Decision Conference (CCDC), 2018.

[26] D. Valério and J. Sá da Costa, "Variable order fractional controllers," Asian Journal of Control, vol. 15, no. 3, pp. 648-657, 2013.

[27] C. A. Monje, Y. Chen, B. M. Vinagre, D. Xue, and V. Feliu-Batlle, Fractional-order systems and controls: fundamentals and applications. London: Springer Science \& Business Media, 2010.

[28] C. F. Lorenzo and T. T. Hartley, "Initialization, conceptualization, and application in the generalized (fractional) calculus," Critical Reviews in Biomedical Engineering, vol. 35, no. 6, pp. 447-553, 2007.

[29] D. Mozyrska and Z. Bartosiewicz, "On Observability of Nonlinear Discrete-Time Fractional-Order Control Systems," in New Trends in Nanotechnology and Fractional Calculus Applications, D. Baleanu, Z. B. Guvenc, and J. A. T. Machado, Eds. Dordrecht: Springer Netherlands, 2010, pp. 305-312.

[30] D. Mozyrska, E. Pawuszewicz, and M. Wyrwas, "Local observability and controllability of nonlinear discrete-time fractional order systems based on their linearisation," International Journal of Systems Science, vol. 48, no. 4, pp. 788-794, Mar. 2017.

[31] D. Simon, Optimal state estimation: Kalman, $H \infty$ and nonlinear approaches. John Wiley \& Sons, 2006.

[32] K. B. Oldham and J. Spanier, The fractional calculus: theory and applications of differentiation and integration to arbitrary order. Mineola, N.Y: Dover Publications, 2006. 